\title{
Grid Computing: The European Data Grid Project
}

\author{
Ben Segal (on behalf of the Project) ${ }^{1}$ \\ CERN, 1211 Geneva 23, Switzerland
}

\begin{abstract}
The goal of the European Data Grid Project is the development of a novel environment to support globally distributed scientific exploration involving multi-PetaByte datasets. The project will devise and develop middleware solutions and testbeds capable of scaling to handle many PetaBytes of distributed data, tens of thousands of resources (processors, disks, etc.), and thousands of simultaneous users. The scale of the problem and the distribution of the resources and user community preclude straightforward replication of the data at different sites, while the aim of providing a general purpose application environment precludes distributing the data using static policies. We will construct this environment by combining and extending newly emerging "Grid" technologies to manage large distributed datasets in addition to computational elements. A consequence of this project will be the emergence of fundamental new modes of scientific exploration, as access to fundamental scientific data is no longer constrained to the producer of that data. While the project focuses on scientific applications such as High Energy Physics, Earth Sciences and Bio-Informatics, issues of sharing data are germane to many applications and thus the project has a potential impact on future industrial and commercial activities.
\end{abstract}

\section{INTRODUCTION}

Substantial investment in R\&D has been made over the past decade in two areas of prime importance for high performance computing: meta-computing, and componentbased clusters. The first of these has its roots firmly in the supercomputing environment where there is typically a single large application with a somewhat fixed parallelisation scheme (fine- or coarse-grained parallelism). The fundamental goal of the meta-computing work has been to provide a mechanism whereby the single large application can execute on a single virtual computer composed of a number of physical supercomputers installed at separate geographic locations. The motivation is that supercomputers are expensive and therefore rare. The maturing work on meta-computing has recently been re-cast in more general terms as the basis of a Computational Grid that will enable a distributed computing infrastructure to be presented as a pervasive, dependable and consistent facility offering unprecedented opportunities for new applications. This vision is thoroughly described in a recent book by Ian Foster and Carl Kesselman [10], which also gives a good summary of the state of the art of meta-computing.

The second area of development that has had a profound effect on large-scale computing is component-based clusters. These are loosely-coupled clusters of computers that can support heterogeneous architectures, a range of network interconnections, a flexible distributed data architecture, and in general provide a (local) infrastructure that can be evolved progressively and seamlessly to accommodate increasing capacity requirements and absorb new technologies. A very early European example is described in [1]. Such developments have been targeted at high-throughput rather than high-performance computing. In other words they are appropriate for embarrassingly parallel applications that can be decomposed to any convenient level of parallel granularity, with the component tasks executing essentially independently. This approach has been used successfully for applications requiring access to very large data collections [11]. The flexibility of the approach has made possible the rapid incorporation of very low cost processing and data storage components developed for the personal computer market [12].

Recent advances in various scientific communities provide a fertile basis for this work. Distributed database research deals with update synchronisation of single transactions on replicas [13]. In [14] Internet specific protocols and worldwide load balancing are discussed. The Globus toolkit [4] provides some of the tools for the construction of computational Grids, but this work has concentrated on the problems of parallel computation-intensive applications, not the dynamic management of large distributed databases. In [15] a design is proposed that extends computational Grids to basic dataintensive Grids. In [16] tertiary storage and cache management are addressed. Our infrastructure will be based on existing frameworks developed by previous and ongoing Grid-related projects, and developed in close collaboration with them. In particular the present project will collaborate directly with key members of the Globus project and with the GriPhyN project [17] that has recently been funded by the United States National Science Foundation. It is expected that a collaboration agreement will be reached with Globus to provide support for key components of the Globus toolkit, and to provide necessary enhancements to deal with scaling and performance issues.

GriPhyN will tackle a similar problem area to that of the current project but over a longer period and with more emphasis on computer science research. The present project has a computer engineering bias focusing on the rapid development of testbeds, trans-national data distribution using a state of the art research network, the demonstration of practical real-life applications, and production quality operation.

\footnotetext{
${ }^{1}$ See Section IV - Acknowledgements.
} 


\section{PROJeCT OUTLINE}

\section{A. Partners}

The main partners in this project are: CERN, INFN (I), CNRS (F), PPARC (UK), NIKHEF (NL), and ESA. Industrial participation is from CSSI (F), DataMat (I) and IBM (UK). Associated institutes from the Czech Republic, Finland, Germany, Hungary, Spain and Sweden are also contributing. The European Commission is funding a substantial portion of the project.

\section{B. Application Areas}

There are three application areas, as follows.

\section{High Energy Physics}

CERN is at present building a new accelerator, the Large Hadron Collider (LHC), that will be the most powerful particle physics accelerator ever constructed when it starts operation in 2005. The computing challenges for LHC are:

- the massive computational capacity required for analysis of the data and

- the volume of data to be processed.

The experiments that will be carried out using the LHC are organised as very large collaborations of research physicists and engineers (1,500-2,000 people on an experiment) employed by hundreds of institutes spread across the 20 European member states and the rest of the world. The computing requirements for LHC will be fulfilled using a large facility at CERN and a number of large regional centres spread across the world in various European countries, North America, and Japan. The Data Grid project will provide a prototype system that could be used as the basis for the initial operation of this distributed computing environment. LHC data analysis will begin as the first data is generated in 2005 and continue for at least ten years, offering opportunities for the deployment of commercial tools based on the work of the project.

\section{Earth Observation Sciences}

The main objective of this part of the Data Grid Project is to bring Grid-aware application concepts into the earth science environment. This will provide a good opportunity to exploit Earth Observation Science (EO) applications that require large computational power and access large data files distributed over a geographically distributed archive.

Specification of EO requirements, their development and implementation is the core of the activity. These components will be validated through small prototyping activities and consolidated by running specific test-beds. A complete application that involves the use of atmospheric Ozone data is selected as a specific test. The scalability of the Grid environment to meet earth science user community requirements will be investigated. This will constitute the basis for the evolution of the testbeds toward much larger computational and data processing requirements.

\section{Bio-Informatics}

The field of Bio-Informatics (also known as Computational Biology) has led to numerous important discoveries and practical applications and is considered as one of today's most promising and expanding scientific fields.

The recent explosion of data acquired by automated gene sequencers and other experimental techniques requires vast amounts of computing power to analyse the biological functions of genes. Additionally thousands of databases contain already collected molecular data, which could be correlated and exploited. Today the Bio-Informatics community lacks the necessary infrastructure to process all this data. Therefore the development of an international infrastructure, which will provide the means of correlating large amounts of data in a transparent way, is of great interest.

In particular we plan to perform:

- Production, analysis and data mining of data produced within projects of sequencing of genomes or in projects with high throughput for the determination of threedimensional macromolecular structures. Comparison between genomes on evolutionary scale.

- Production, storage, comparison and retrieval of measures of the genetic expression levels obtained through systems of gene profiling based on micro-arrays, or through techniques that involve the massive production of nontextual data as still images or video.

- Retrieval and in-depth analysis of the biological literature (commercial and public) with the aim of the development of a search engine for relations between biological entities.

\section{Project Structure}

The project structure is as follows.

\section{Workload Scheduling and Management}

The innovative issues to be tackled by the workload management section of the project result from the following factors: the dynamic relocation of data, the very large numbers of schedulable components in the system (computers and files), the large number of simultaneous users submitting work to the system, and the different access policies applied at different sites and in different countries.

Workload management must develop strategies for planning job decomposition and task distribution based on knowledge of the availability and proximity of computational capacity and the required data. Job description languages based on previous work will be extended as necessary to express data dependencies. In the general case it will be necessary to compare different decomposition and allocation strategies to take account of the availability of the data in more than one location (either in the form of replicas, or in the data cache), and the commitment level of the computational capacity at the different sites. This involves making relative cost estimates that may be complicated by the need to take account of factors like potential execution delays (e.g. the time a task spends queuing in an over-committed system), the generation of new cache copies at under-committed sites, and the potential delays 
incurred by data migration between secondary and tertiary storage.

The general-purpose nature of the Grid implies an unpredictable, chaotic workload generated by relatively large numbers (thousands) of independent users - again in contrast to the supercomputing environments that have been the target of most previous meta-computing projects.

The complications of dealing with the inhomogeneous resources of the general-purpose data-intensive Grid will require new approaches to co-allocation and advance reservation, and to recovery strategies in the event of failures of components.

\section{Data Management}

The project will develop and demonstrate the necessary middleware to permit the secure access of massive amounts of data in a universal global name space, to move and replicate data at high speed from one geographical site to another, and to manage the synchronisation of remote data copies. Novel software will be developed such that strategies for automated wide-area data caching and distribution will adapt according to dynamic usage patterns. It will be necessary to develop a generic interface to the different mass storage management systems in use at different sites, in order to enable seamless and efficient integration of distributed storage resources. Several important performance and reliability issues associated with the use of tertiary storage will be addressed.

\section{Grid Monitoring Services}

The results of this activity will enable monitoring of the use of geographically distributed resources on a scale and with a transparency not previously available. It will be possible to assess the interplay between computer fabrics, networking and mass storage in supporting the execution of end-user Grid applications to a level of detail beyond that currently possible in highly distributed environments.

New instrumentation APIs will be defined to provide data on the performance and status of computing fabrics, networks and mass storage. These will be supported by new local monitoring tools developed to provide appropriate levels of detail on performance and availability to support workload and data management scheduling strategies, and also to enable the study of individual application performance. The highly distributed nature and large scale of the proposed Grid will require the development of new methods for short and long term storage of monitoring information to enable both archiving and near real-time analysis functions, and the development of new effective means of visual presentation of the multivariate data.

\section{Local Fabric Management}

The Grid issues for the local fabric management are related to the support of information publication concerning resource availability and performance, and the mapping of authentication and resource allocation mechanisms to the local environment. Innovative work will be required, to provide flexible and resilient management of a very large local fabric. The existence of the Grid adds a new level of complexity to dynamic configuration changes and error recovery strategies, requiring pre-determined policies and full automation. The Grid must operate in a self-describing way, using scheduling algorithms that adapt to the availability of resources and current performance characteristics. Comparable strategies will be developed for the management of the local computing fabrics. However, these strategies cannot simply be copied from previous Grid-related work as they have very different scaling characteristics and timing constraints. The local fabric must deal with tens of thousands of components. The existing Grid technologies deal with a structured environment consisting of a relatively small number of nodes (geographical locations) each of which is described in terms that aggregate the definition and status of the local resources into a restricted number of parameters. The approach to the management of the local fabric will have more in common with industrial control systems than traditional computer system management systems. The latter do not scale to very large configurations and the fault-tolerant techniques in general use imply substantial investments in redundant hardware. The innovative approach to be used in this project (self-healing) is to develop algorithms that will enable faults to be detected and isolated, automatically reconfiguring the fabric and re-running the tasks. Similarly new and updated components will announce themselves to the system and be incorporated automatically into the fabric.

\section{Mass Storage Management}

Particle physics has decades of experience of large-scale data handling. Typically this has involved shipping data on magnetic tapes between countries although recently significant volumes are transferred by the Internet. All of the partners have experience of handling data from several different accelerator centres and the incompatibilities that this entails. This task proposes to introduce standards for handling LHC data that, if successful, should be adopted by others in the field. Since many of the partners run multi-disciplinary data centres, the benefits should be available to other fields who deal with large-scale data processing.

The Mass Storage Management activity will ensure the development of a uniform interface to the very different systems used at different sites, to provide interchange of data and meta-data between sites, and to develop appropriate resource allocation and information publishing functions to support the Grid.

\section{The Testbed, Network \& Applications}

The fundamental goal is to demonstrate that we can build very large clusters from inexpensive mass market components and integrate these clusters into a coherent data intensive Grid. This approach towards commodity-based solutions has been successfully applied by most of the partners over the past ten years. Anticipating and fostering the move to Linux and PCs, these have now been successfully integrated into the production environments of the partners. Also the community to which several of the partners belong, High-Energy Physics, 
has a long experience of exploiting leading edge high bandwidth research networking.

The project will place a major emphasis on providing production quality testbeds, using real-world applications with real data drawn primarily from two scientific areas - highenergy physics and earth observation. These areas offer complementary data models that allow the demonstration of different aspects of the Data Grid. The High-Energy Physics model is characterised by the generalised dynamic distribution of data across the Grid including replica and cache management. In contrast, earth observation applications will demonstrate uniform access to large distributed databases.

The extension of the project to include biological sciences is motivated by the opportunity that the Data Grid offers as a catalyst for cross-border integration of national testbeds and databases used by the biological sciences. Technical requirements that will have to be met for these applications include support for updating of databases, and strict security for data arising from the commercial interests present in the area of biological sciences.

In addition to providing a demonstration environment, the testbed must be operated as a production facility for real applications with real data. This is the only way to ensure that the output of the R\&D programme in terms of models, principles and software fulfils the basic requirements for performance and reliability and has practical application to real-world computing.

\section{HEP APPLICATIONS AND REGIONAL CENTRES}

The High Energy Physics (HEP) community has the need of sharing information, very large databases (several PetaBytes) and large computational resources (thousands of fast PC's) throughout its centres distributed across Europe, and in several other countries in the other continents. One of the main concerns of the HEP community is to improve the efficiency and speed of their data analysis by integrating the processing power and data storage systems available at the separate sites.

HEP is today organised in large experimental collaborations - 1,600 or more researchers coming from countries all around the world for the larger ones. Four subtasks in this project correspond to the four experiments (ALICE, ATLAS, CMS, LHCb) in preparation for the CERN Large Hadron Collider (LHC) due to enter operation in 2005. Each experiment creates its own data set and all the researchers participating to one experiment have access to, and analyse, the same data. The data sets are different for the four experiments, reflecting the difference in the experimental setups and in the physics goals. However they are similar in nature and also the basic access and processing requirements are essentially the same. Therefore the work of the Data Grid project (particularly at the middleware and interface levels) is equally applicable to all of them.

The four LHC experiments will produce several PB of original (also called raw) data per year. In particular ATLAS and CMS foresee to produce $1 \mathrm{~PB} /$ year of raw data and $200 \mathrm{~TB}$ of
Event Summary Data (ESD) resulting from the first reconstruction pass. Analysis data will be reduced to a few tens of TB, and event tag data (short event indexes) will be a few TB. ALICE foresees to acquire 2PB/year of raw data, combining the heavy ion data with the proton data. $\mathrm{LHCb}$ will be generating $\sim 4 \mathrm{~PB} /$ year of data covering all stages of raw and MC processing. For typical analysis a subset of raw data should be sufficient, but the full ESD sample may be needed, at least in the initial phases of the experiment. The computing power required to analyse these data is estimated in the range of thousands of SpecInt95.

The raw data are generated at a single location where the experiments are run, CERN, but the sheer computational capacity required to analyse them implies that the analysis must be performed at geographically distributed centres. The storage and CPU capacity available at a single location is expected to be inadequate for the amount of the data to be traversed. To obtain the necessary resources and ensure an acceptable ergonomy of use, distributed processing will be exploited on resources situated both locally and remotely. Therefore each of the experiments will need to exploit a world-wide distributed data base and computing hardware comprising:

- very large amounts (many PetaBytes) of distributed data,

- very large numbers (tens of thousands) of computing resources,

- $\quad$ and thousands of simultaneous users.

This scale requires the innovative use of large scale distributed computing resources and mass storage management systems to organise a hierarchical distribution of the data between random access secondary storage (disk) and serial tertiary storage (such as magnetic tape). The data transfer requires high-speed point-to-point replication facilities and a system that checks and maintains data consistency. Duplicated data should be used to help balance the search and network loads according to the response of the site. Consequently the computing model adopted by the LHC collaborations is distributed and it will be implemented via a hierarchy of Regional Centres.

Different Regional Centres will focus on specific functionality and tasks. Some will specialise on simulation whereas others will take part in specific reconstruction tasks. In general raw event data are stored at CERN and only particular subsets of the event data are stored at the centres. The scale of the problem precludes straightforward replication of all data at different sites, while the aim of providing a general-purpose application environment precludes distributing the data using static policies. The computing model will have to handle generalised dynamic distribution of data including replica and cache management. HEP user requirements can be summarised as follows:

- All physicists should have, at least in principle similar access to the data, irrespective of their location.

- Access to the data should be transparent and as efficient as possible and the same should hold for the access to the computational resources needed to process the data.

The HEP Grid application tasks will be conducted as part of the computing projects of the four LHC experiments. These 


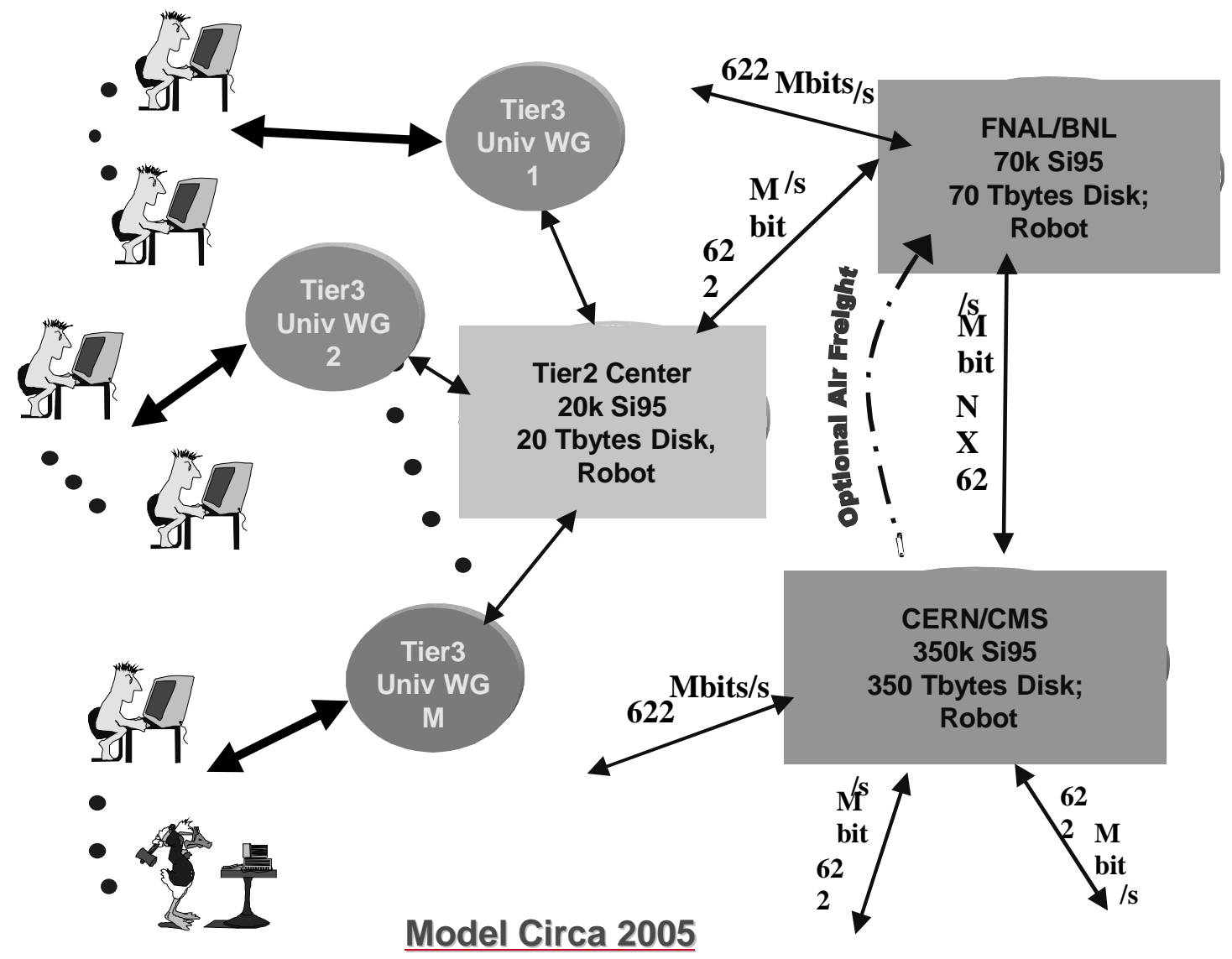

experiments are now developing their computing infrastructure, and in particular their reconstruction and analysis programs. The realisation of this complex of interrelated data samples stored at different centres and accessible online both locally and remotely constitutes a formidable technical and organisational challenge. Smooth operation of the whole system will be mandatory when real data will begin to flow from the apparatus in order to produce reliable physics results in a timely fashion. To enable this development, and at the same time continuously monitor the feasibility of the physics objectives, the four experiments have planned from now to 2005 large-scale tests called Data Challenges. These have the objective to assess the development of the software and of the distributed computing model, at least for Tier 0 and 1 centres.

In the Data Challenges, large samples of data will be simulated via very advanced simulation programs. These data are then analysed pretending that they are coming from the real experiment. The programs to perform these analyses are already partially developed. This is a major exercise involving several tens of institutes and hundreds of physicists, and the four experiments are planning to conduct it exploiting the tools and services provided by our project. A large part of the project's value comes from real users performing large-scale experimentation and benefits from a very large community that is preparing a world-wide distributing computing infrastructure needed for its experimental programme.
The two larger LHC experiments, ATLAS and CMS, have a substantial participation from US laboratories. In the US also it has been realised that HEP applications are ideal Grid test cases, and various Grid projects related to the LHC are already in an advanced phase (GriPhyN, China Clipper, PPDG). Collaboration with these projects is most important for the experiments and is already starting, based on the working relations between members of the same experiments and on the need to share data and computational resources across the whole experiment community. It is therefore expected that several physicists from the US and Japan will participate in Data Challenges and add to the global scope of the project. 


\section{ACKNOWLEDGMENTS}

This paper is adapted from proposal documents prepared by the European Data Grid Project for submission to the European Commission. Acknowledgements are due to many project collaborators, in particular the Project Manager Fabrizio Gagliardi/CERN, the members and alternate members of the Project Management Board ${ }^{2}$, the Work Package Managers ${ }^{3}$ and to Mark Parsons/EPCC (Edinburgh) for overall help with the proposal.

\section{REFERENCES}

[1] J-P.Baud et al, SHIFT, The Scalable Heterogeneous Integrated Facility for HEP Computing, Proc. Conference on Computing in High Energy Physics - March 1991 - Tsukuba, Japan - Universal Academic Press 1991

[2] J. Apostolakis et al., General-purpose parallel computing in a High-Energy Physics experiment at CERN, High Performance Computing and Networking (Lecture Notes in Computer Science no. 1067), Brussels 1996 - Springer, Berlin, 1996

[3] M.Lamanna, The Compass Computing Farm Project, Proc. Computing in High Energy Physics - February 2000 - Padova, Italy - Imprimenda 2000

[4] I.Foster and C.Kesselman, The Globus Project - a Progress Report, Proc. Heterogeneous Computing Workshop, Los Alamitos, CA - IEEE Computer Society Press 1998

[5] Architectural Support for Extensibility and Autonomy in Wide-Area Distributed Object Systems, Tech.Report CS-98-12, Computer Science Dept., Univ. of Virginia, Charlottesville, VA, 1998

[6] M. Romberg, The UNICORE Architecture: Seamless Access to Distributed Resources, Proc. Eighth IEEE International Symposium on 'High Performance Distributed Computing' HPDC -August 1999 - IEEE Computer Society, Los Alamitos, CA, 1999

[7] D.Roweth, P.Fuhrmann, M.GastHuber, Eurostore - Design and First Results, Proc. 16 ${ }^{\text {th }}$ IEEE Symposium on Mass Storage Systems - San Diego, March 1999 - IEEE 199

[8] H.Newman, L.Perini et al, Models of Networked Analysis at Regional Centres for LHC Experiments, CERN/LCB 2000-01, CERN, Geneva, Switzerland, 2000

[9] Catalogues for molecular biology and genetics:

DBCAT, The Public Catalog of Databases: http://www.infobiogen.fr/services/dbcat

The Biocatalog (catalogue of software): http://corba.ebi.ac.uk/Biocatalog/biocatalog_ebi.html

[10] I.Foster, C.Kesselman (editors), The Grid - Blueprint for a New Computing Infrastructure, Morgan Kaufman, San Francisco, 1999

[11] J.D.Shiers, Massive-Scale Data Management using Standards-Based Solutions, Proc. 16th IEEE Symposium on Mass Storage Systems - IEEE Computer Society 1999

[12] The Beowulf Project: http://www.beowulf.org/

[13] Y. Breitbart et al, Update Propagation Protocols For Replicated Databases, SIGMOD Conference 1999

[14] M. Rabinovich, Caching and Replication on the Internet, Tutorial, VLDB Conference 1998

[15] I. Foster, C. Kesselman et al, The Data Grid: Towards an Architecture for the Distributed Management and Analysis of Large Scientific Datasets, Network Storage Symposium, 1999

[16] A. Shoshani et al, Multidimensional Indexing and Query Coordination for Tertiary Storage Management, SSDBM 1999

[17] GriPhyN- Grid Physics Network - http://www.phys.ufl.edu/ avery/mre/

2 Project Management Board members and alternate members are: Hans Falk Hoffmann/CERN, Les Robertson/CERN, Paul Jeffreys/PPARC, Robin Middleton/PPARC, Federico Ruggieri/INFN, Mirco Mazzucato/INFN, Guy Wormser/CNRS, Francois Etienne/CNRS, Luigi Fusco/ESA, Gabriele Brugnoni/ESA, Kors Bos/NIKHEF, Arjen van Rijn/NIKHEF.

3 Work Package Managers (if not mentioned above) are: Cristina Vistoli/INFN, Tim Smith/CERN, John Gordon/RAL, Christian Michau/CNRS, Federico Carminati/CERN, Gianfranco Mascari/CNR. 